craniocervical and systemic, should be considered in pediatric stroke when fibromuscular dysplasia is suspected. In a case-report of a 12-year-old boy with ischemic stroke caused by intracranial fibromuscular dysplasia, preliminary imaging investigations included CT and MR angiography, but a definitive diagnosis was reached following a conventional cerebral angiogram (Shea KJ, et al. Pediatr Neurol 2011 Mar;44(3):214-7).

\title{
MYOPATHIES
}

\section{CONGENITAL RYR1-ASSOCIATED MYOPATHIES}

Investigators from the Children's Hospital of Philadelphia, NINDS, and other centers in the US and France, report a series of 11 patients with severe neonatal RYR1associated myopathy confirmed by genetic testing. Clinical features included decreased fetal movements, hypotonia, poor feeding, respiratory impairment, arthrogryposis, ophthalmoplegia, and femur fracture and hip dislocation at birth. RYR1 mutations were dominant in 4 patients and recessive in 7. Muscle ultrasound in 6 patients showed relative sparing of the rectus femoris muscle. All patients with dominant mutations had classic central cores on muscle biopsy; patients with recessive mutations showed histologic heterogeneity. (Bharucha-Goebel DX, Santi M, Medne L, et al. Severe congenital RYR1associated myopathy. The expanding clinicopathologic and genetic spectrum. Neurology 2013 Apr 23;80(17):1584-9). (Resp.: Dr Bonnermann. Carsten.bonnermann@nih.gov).

COMMENT. Classic central core disease (CCD) due to mutations in the RYR1 gene typically presents with mild to moderate hypotonia, developmental delay, proximal muscle weakness, and occasional hip dislocation. The present series of patients has a more severe neonatal RYR1-associated myopathy caused by both dominant and recessive mutations of the gene and expands the clinical spectrum of central core disease. Sparing of the rectus femoris muscle on ultrasound should prompt evaluation for RYR1associated myopathy.

Subtypes of congenital myopathies in UK. Of 54 patients with muscle biopsies available, diagnosed over a 5-year period at Great Ormond Street Hospital for Children, London, 29 (54\%) had a core myopathy (central core disease, multi-minicore disease), 9 (17\%) had nemaline myopathy, 7 (13\%) had myotubular/centronuclear myopathy, 2 (4\%) had congenital fibre type disproportion, $6(11 \%)$ had isolated type 1 predominance and 1 (2\%) a mixed core-rod myopathy. Of 44 with a genetic diagnosis, RYR1 was mutated in $26(59 \%)$. The genetic defect was unidentified in 1/3 of congenital myopathies (Maggi L, Scoto M, Cirak S, et al. Neuromuscul Disord 2013 Mar;23(3):195-205).

\section{RYR1 MUTATIONS, EXERTIONAL MYALGIA AND RHABDOMYOLYSIS}

Investigators at Guy's \& St Thomas' Hospital, London, UK, and other centers sequenced RYR1 in 39 unrelated families with rhabdomyolysis and/or exertional myalgia and identified 9 heterozygous RYR1 mutations in 14 families, 5 of them previously associated with malignant hyperthermia (MH). Index cases presented from 3 to 45 years 